Accepted Manuscript

Surface Sensitivity of Ultrasonically Treated Carbon Nanotube Network towards Ammonia

Petro M. Lutsyk, Prabakaran Shankar, Alex G. Rozhin , Sergei A. Kulinich

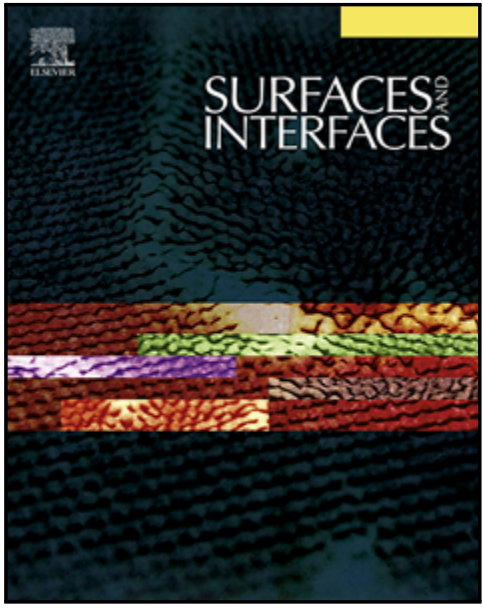

PII:

DOI:

Article Number:

Reference:

To appear in:

Received date:

Accepted date:
S2468-0230(19)30328-1

https://doi.org/10.1016/j.surfin.2019.100363

100363

SURFIN 100363

\section{Surfaces and Interfaces}

12 June 2019

21 July 2019

Please cite this article as: Petro M. Lutsyk, Prabakaran Shankar, Alex G. Rozhin , Sergei A. Kulinich, Surface Sensitivity of Ultrasonically Treated Carbon Nanotube Network towards Ammonia, Surfaces and Interfaces (2019), doi: https://doi.org/10.1016/j.surfin.2019.100363

This is a PDF file of an unedited manuscript that has been accepted for publication. As a service to our customers we are providing this early version of the manuscript. The manuscript will undergo copyediting, typesetting, and review of the resulting proof before it is published in its final form. Please note that during the production process errors may be discovered which could affect the content, and all legal disclaimers that apply to the journal pertain. 


\title{
Surface Sensitivity of Ultrasonically Treated Carbon Nanotube Network towards Ammonia
}

\author{
Petro M. Lutsyk ${ }^{1}$, Prabakaran Shankar ${ }^{2,3}$, Alex G. Rozhin ${ }^{1}$, Sergei A. Kulinich ${ }^{2,4,5, *}$ \\ ${ }^{1}$ School of Engineering and Applied Science, Aston University, Aston Triangle, B4 7ET Birmingham, UK \\ ${ }^{2}$ Institute of Innovative Science and Technology, Tokai University, Hiratsuka, 2591292 Kanagawa, Japan \\ ${ }^{3}$ Biological \& Nanoscale Materials Lab, School of Advanced Materials Science \& Engineering, \\ Sungkyunkwan University (SKKU), Suwon 16419, Korea \\ ${ }^{4}$ Research Institute of Science and Technology, Tokai University, Hiratsuka, 2591292 Kanagawa, Japan \\ ${ }^{4}$ Department of Mechanical Engineering, Tokai University, Hiratsuka, 2591292 Kanagawa, Japan
}

\begin{abstract}
Detection of ammonia by carbon nanotubes is an extensively studied area where tremendous progress was achieved so far in their sensing performance. In this paper, we focused on carbon nanotube network sensors of $\mathrm{NH}_{3}$ aiming to develop better understanding of their gas detection behavior and improved sensing response. Our experiments showed improved sensing performance for single-walled carbon nanotubes of $(6,5)$ chirality ultrasonically treated at high power and then forming bundled nanotube network upon thermal annealing. Two chemiresistive sensing pathways were observed for such networks resulting in increased and decreased resistance in presence of different ammonia concentrations from $10^{2}$ to $10^{4} \mathrm{ppm}$. This multidirectional chemiresistive response was comprehensively explained via various phenomena, such as partial and full neutralization of $p$-type conductivity of the nanotubes, change of the Schottky barrier, and dipoles at the interface between nanotubes and gold electrodes. The proposed sensing mechanisms are believed to provide strong support for further development of ammonia sensors with optimized performance.
\end{abstract}

Keywords: Carbon nanotubes; Bundled nanotube networks; Ammonia; Sensing

* Corresponding authors. Email: skulinich@tokai-u.jp (S.A. Kulinich)

Page 1 of 15 


\section{Introduction}

The exceptional electrical properties of carbon nanotubes (CNTs) have resulted in development of various nano-scaled electronic devices, chemical sensors, electron field emission sources, scanning probes and actuators, and so on [1]. However, preparation procedures and/or treatment of CNTs are well-known to affect their electrical properties; for example, treatment of single-walled carbon nanotubes (SWNTs) with oxidizing agents increases their conductivity [2]. In general, metallic or semiconducting nature of SWNTs strongly depends on their chirality and dopants [3-6]. Additionally, the van der Waals interaction between SWNTs results in the formation of their bundles, which improves charge transport properties. According to the percolation theory, isolated nanotube networks and bundled carbon nanotube networks (BNNs) can be metallic and/or semiconducting depending on the network geometry [3,7]. Furthermore, surface properties of isolated nanotubes and BNNs can vary owing to disorders and defect sites [3,5,7]. Therefore, in-depth studies of physico-chemical impact of surrounding gas molecules on the behavior of CNT networks are essential to develop any devices on their basis.

Since the discovery of gas sensing properties demonstrated by CNTs [4], research community focused on development of various CNT-based chemical and biological sensors [1]. As a result, selective detection of $\mathrm{NH}_{3}$ by SWNTs is one of the most studied areas with tremendous progress in sensing performance achieved by now [5,8-11]. For example, as a proof of concept, the $\mathrm{NH}_{3}$ gas sensing performance of defectinduced SWNT-based sensors was recently demonstrated to be higher than that of defect-free SWNTs [9]. Preferential adsorption of ammonia molecules on defect sites of SWNTs was suggested as an appropriate gas sensing mechanism in defect-induced SWNT-based sensors [10]. Therefore, controlled incorporation of topological defects to SWNTs can be used to enhance their sensitivity to a variety of chemical vapors [5]. In turn, sensors based on multi-walled CNTs revealed that adsorption of $\mathrm{NH}_{3}$ molecules is related to the nature of nanotubes and to defects inside [11].

Regarding CNT bundles (or BNNs), the influence of network geometry and morphology [3,12], nanotube doping [7], and bundle length [13] on their electrical transport properties was investigated to date. However, studies of both charge transfer by gas molecules and surface sensitivity for bundled nanotubes are necessary to develop more efficient chemical sensors, as well as field-effect transistors made of them. Although substantial advancements in the nanotube-based sensors have been made [4-6,9-11,14-16], the details of $\mathrm{NH}_{3}$ sensing mechanisms still remain substantially uncertain $[8,14]$. In this paper, we focused on studying the surface sensitivity of highly-semiconducting isolated nanotubes and BNNs. Our particular target was to understand better $\mathrm{NH}_{3}$ sensing mechanisms of BNNs with high content of semiconducting CNTs and with defects introduced by means of ultrasonic treatment. 


\section{Materials and methods}

\subsection{Materials}

Purified SWNTs (SG65 CoMoCAT from SWeNT, Inc.) were used as a starting material to prepare the SWNT dispersions. As a dispersing agent, we used poly[(9,9-dioctylfluorenyl-2,7-diyl)-alt-co-(6,6'-\{2,2'bipyridine\})] (PFO-BPy) purchased from American Dye Source Inc.

\subsection{Preparation of SWNT dispersions}

CNT powder (3 mg) was dispersed in $10 \mathrm{~mL}$ of toluene in presence of $20 \mathrm{mg}$ of PFO-BPy. Tip ultrasonication was performed by a UP400S ultrasonic processor (Hielscher Ultrasonics $\mathrm{GmbH}$ ) for $16 \mathrm{~h}$, with its pulse length being $75 \%$. To induce different level of defects, we used two regimes for ultrasonic power, 20 and $60 \%$ of maximum, to obtain samples S1 and S2, respectively (Fig. 1). The produced SWNT dispersions were centrifuged for $30 \mathrm{~min}$ with an Optima Max-XP ultracentrifuge (Beckman Coulter) at 30,000 rpm (MLS 50 rotor) to remove nanotube bundles and to get well purified supernatant dispersions with high concentration of semiconducting SWNTs. According to the literature, ultrasonication of SWNT powders in presence of PFO-BPy results in a highly selective dispersion of SWNTs with $(6,5)$ chirality $[17,18]$. Other chiralities and non-chiral CNTs are known to be removed during such a procedure, leading to dispersions with a high content $(>99 \%)$ of semiconductor nanotubes $[17,18]$.

\subsection{Preparation of isolated carbon nanotubes and BNNs}

The as-prepared highly purified dispersions of SWNTs were dropcast onto commercial interdigitated electrodes (G-IDEAU10, from DropSens). Equal volume $(100 \mu \mathrm{L})$ of samples $\mathrm{S} 1$ and $\mathrm{S} 2$ was coated drop by drop onto the electrodes and simultaneously dried at $40{ }^{\circ} \mathrm{C}$ (Fig. 1). The obtained film-like samples were then annealed at $300{ }^{\circ} \mathrm{C}$ for $2 \mathrm{~h}$. The annealing is known to lead to polymer decomposition and formation of more tightly packed bundles [19,20]. Upon annealing, the film-like samples S1 and S2 gave rise to BNNs which were labelled as samples B1 and B2, respectively (Fig. 1). For comparison, similar samples were also prepared on quartz substrates, which upon annealing were then used for optical studies.

Page 3 of 15 


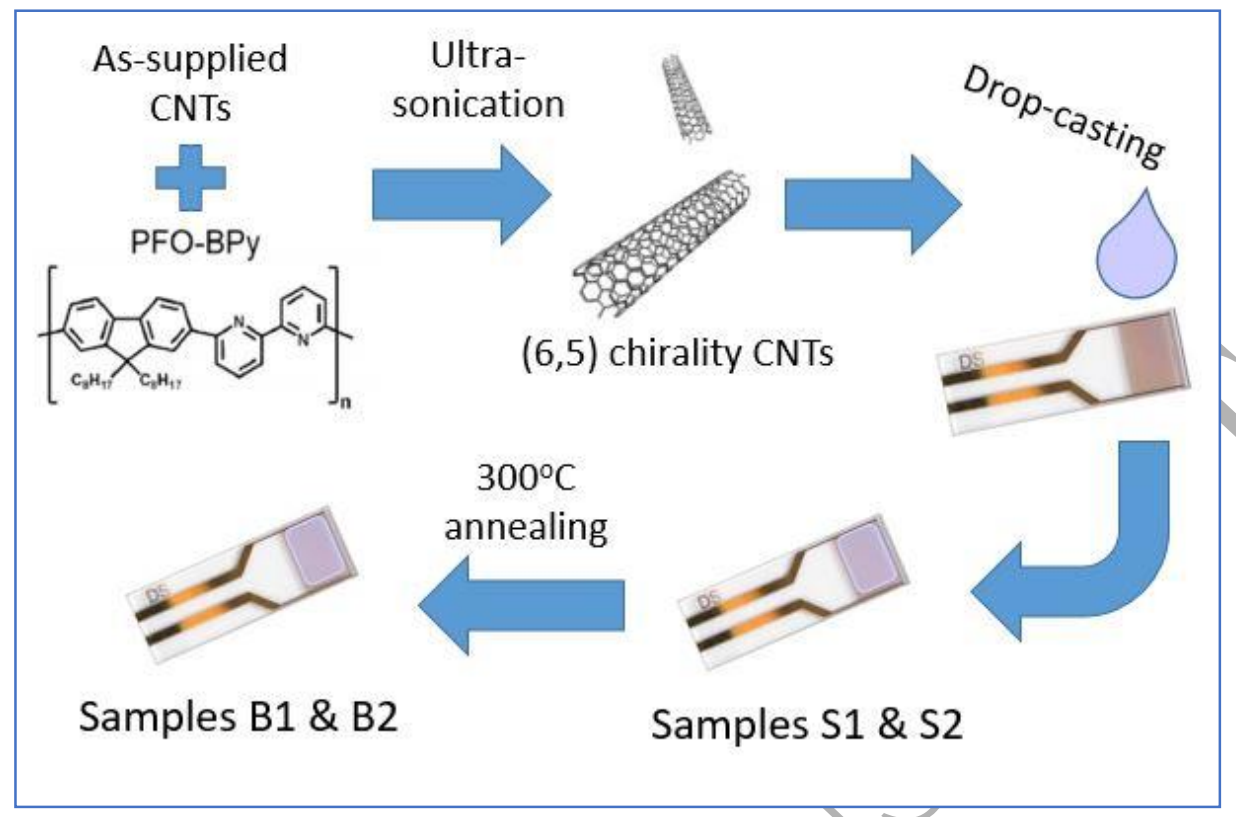

Figure 1. Schematic description of procedures used to prepare samples. At first, CNT dispersion was mixed with PFO-BPy and ultrasonicated at higher and lower power to produce CNT dispersions with high and low defect level (both being mainly of $(6,5)$ chirality). Then the two dispersions were drop-cast onto interdigitated electrodes to give rise to samples S1 and S2 (with low and high level of defects). Finally, they were annealed at $300^{\circ} \mathrm{C}$, resulting in samples $B 1$ and $B 2$.

\subsection{Material characterization}

The absorption spectra were measured with a Lambda 1050 UV/VIS/NIR spectrometer (Perkin Elmer). The maps of PL excitation-emission, PLE maps (where the X-axis is the wavelength of PL emission, $\lambda_{\mathrm{EM}}$, and the $\mathrm{Y}$-axis is the wayelength of PL excitation, $\lambda_{\mathrm{EX}}$ ) were measured by a NanoLog excitation-emission spectrofluorometer (Horiba). The spectrofluorometer was equipped with an InGaAs array detector for nearinfrared range measurements cooled with liquid nitrogen.

\subsection{Sensing characterization}

The electrical and sensing properties of isolated nanotube (S1 and S2) and BNN (B1 and B2) samples were observed using a digital multimeter from Fluke (model 8845A) with two-probe configuration. Gas sensing experiments were carried out using a commercial chamber from Figaro (bench-top test chamber, model SR-3) where film-like samples drop-cast onto interdigitated electrodes were placed and connected to chamber's electrical feedthrough. The surface sensitivity of the samples was analyzed using volatile organic 
compounds (VOCs), such as acetone, ammonia, ethanol, methanol, and isopropanol. The static gas-liquid distribution method previously described elsewhere [21] was used to estimate the volume of VOC for the desired concentrations of vapor. The sensing studies were carried out at room temperature $(295 \mathrm{~K})$ with $(45 \pm 3) \% \mathrm{RH}$. The sensing response, $S$, was calculated according to the below formula:

$$
S=\frac{R_{g}-R_{a}}{R_{a}} \times 100 \%
$$

where $R_{a}$ is the sample resistance in the ambient atmosphere and $R_{g}$ is its resistance in specifie gas/vapor atmosphere.

\section{Results and discussion}

\subsection{Optical characterization}

\subsubsection{Dispersions of SWNTs}

The absorption spectra of the SWNT+PFO-BPy dispersion obtained at lower sonication power (sample S1) are seen in Fig. 2a to have very strong and narrow peaks at 999 and 573 nm, which are assigned to the $E_{11}$ and $E_{22}$ transitions for isolated SWNTs with the (6,5) chirality, respectively (Fig. 2a, curve 1). The spectra of sample S2 obtained at higher sonication power have absorption peaks at 1,000 and $573 \mathrm{~nm}$ demonstrating a small red shift of the $\mathrm{E}_{11}$ transition, which may be caused by stronger sonication of the CNTs comprising this sample. To demonstrate the effect of PFO-BPy dispersing agent on selective separation of the $(6,5)$ chirality, Fig. 2a compares spectra of samples S1 and S2 (curves 1 and 2) with a similarly measured absorption spectrum of the same SWNTs dispersed with sodium dodecyl-benzene sulfonate (SDBS, curve 3) as a non-selective dispersing agent. The detailed procedure for obtaining SWNTSDBS dispersions is described elsewhere [22]. Comparison of the spectra for samples S1 and S2 (curves 1 and 2 in Fig. 2a) and that of reference SWNT-SDBS dispersion (curve 3 in Fig. 2a) shows a strong decrease in the background absorption of samples S1 and S2, implying very efficient removal of metallic CNTs, which is in good agreement with previous work [17,18]. Importantly, curves 1 and 2 exhibit almost no absorption in the range of $400-500 \mathrm{~nm}$ (where CNTs with metallic properties absorb). Thus, during ultrasonic treatment, SWNT dispersions with a high content of semiconductor phase with $(6,5)$ chirality were separated, while the fraction of metallic CNTs was significantly reduced. 
(a)

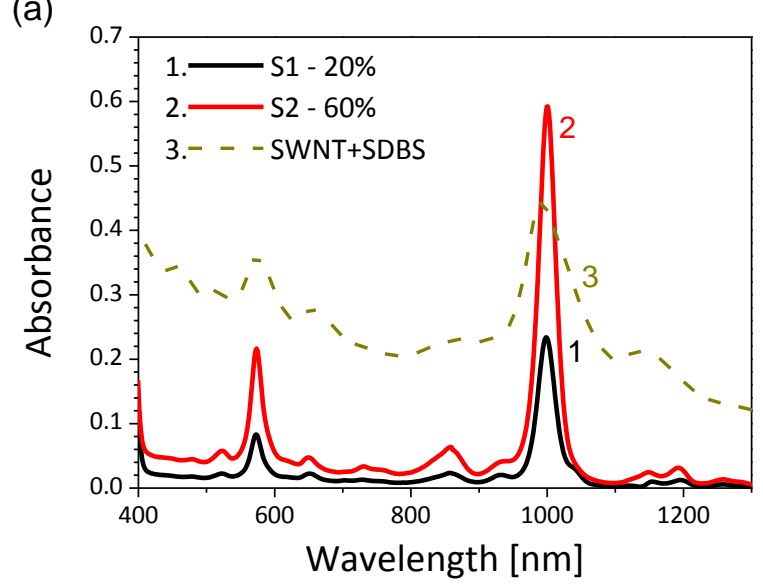

(c)

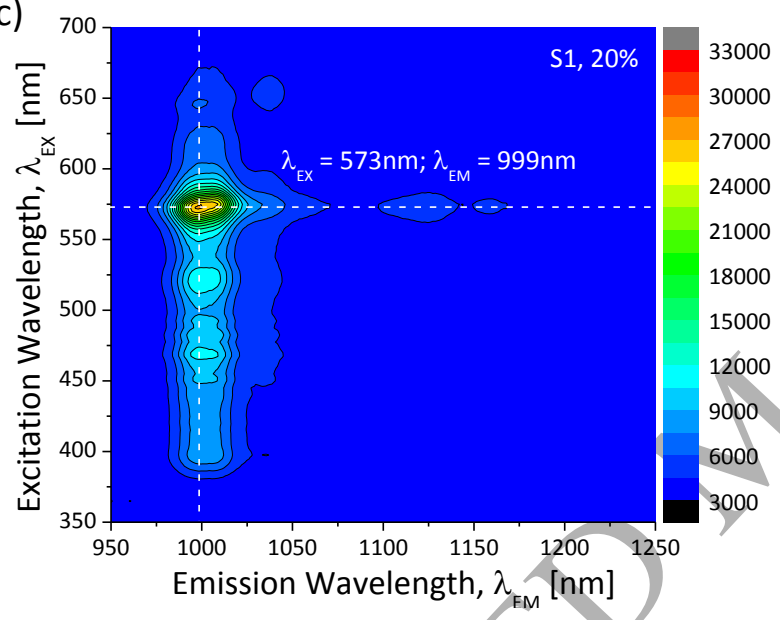

(b)

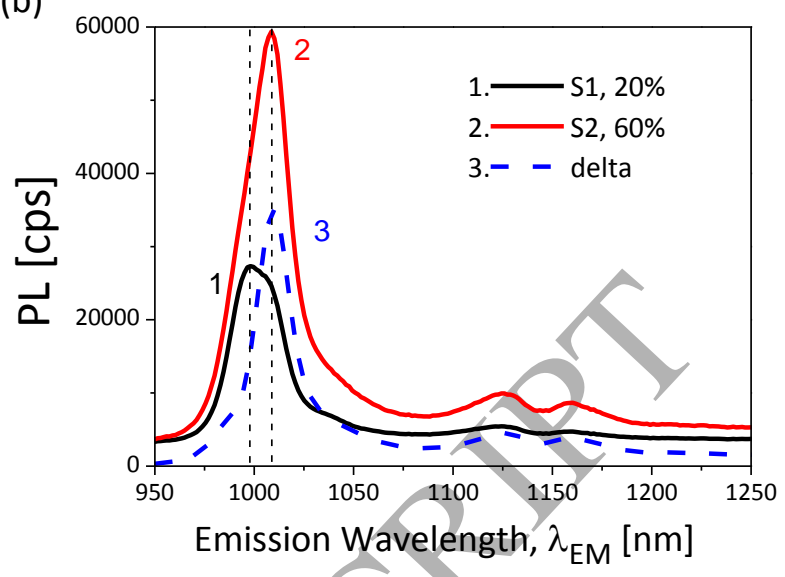

(d)

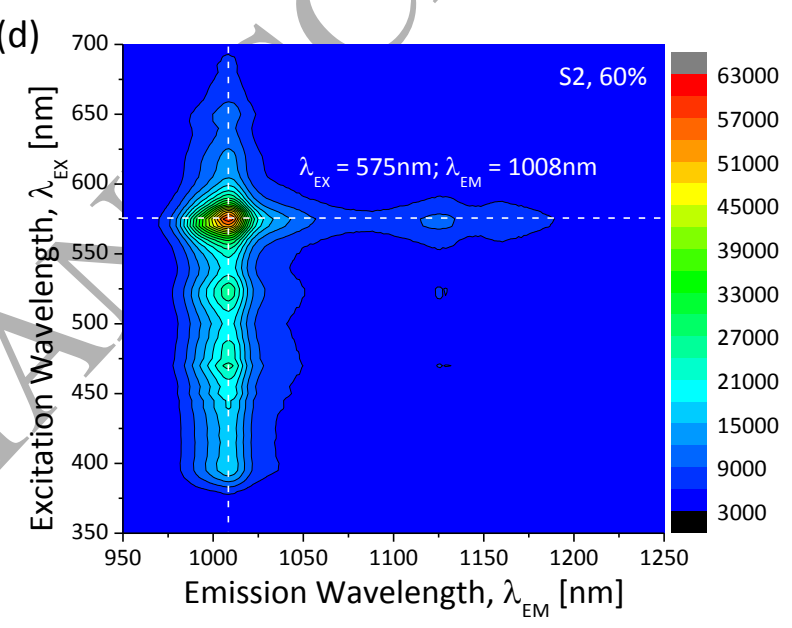

Figure 2. (a) Absorption spectra of samples S1 (curve 1), S2 (curve 2) measured as dispersions and of SWNT-SDBS dispersion (curve 3) used as reference [22]. (b) PL spectra of samples $S 1$ (curve $1 ; \lambda_{E X}=573 \mathrm{~nm}$ ) and S2 (curve 2; $\lambda_{E X}=575 \mathrm{~nm}$ ) analyzed as dispersions; curve 3 is the difference between curve 2 and curve 1 . (c,d) PLE maps of samples S1 (c) and S2 (d), with 3.5-nm-wide entrance/exit slits being used in monochromator.

The PL spectra of the SWNT+PFO-BPy dispersion sonicated at lower sonication power (S1) are seen in Fig. $2 \mathrm{~b}$ to have a maximum at $\lambda_{\mathrm{EM}}=999 \mathrm{~nm}$ (curve 1), whereas the PL spectra for sample S2 obtained at higher sonication power have a peak at $\lambda_{\mathrm{EM}}=1,008 \mathrm{~nm}$ (Fig. 2b, curve 2). Thus, there is a strong red shift of the PL peak associated with the $\mathrm{E}_{11}$ emission of the CNTs with $(6,5)$ chirality sonicated at higher power (sample S2). To analyze the observed redshift of PL peak, we obtained the differential PL spectrum (Fig. 2b, curve 3), i.e. the difference between curve 2 and curve 1 in Fig. 2b. The differential PL spectrum has a maximum at $1,010 \mathrm{~nm}$, showing predominant growth of the PL emission band peaking at 1,010 nm at high- 
power sonication. This band can be attributed to defected nanotube emission, such as $\mathrm{sp}^{3}$-hybridized carbon [17]. The formation of such defects on SWNTs caused by more energetic sonication was confirmed not only by the red shift of PL maxima, but also by a larger G+/2D peak area ratio in Raman spectra previously reported by Graf and co-workers [17]. The high G+/2D ratio is known to be indicative of defects or impurities in nanotubes [24]. Thus, as expected, more defects were generated in sample S2 sonicated at a higher power.

The PLE maps of the same samples S1 and S2 are presented in Fig. 2c and 2d, respectively, The maps not only allow us to analyze spectral features of the PL emission, but also show the dependence of PL intensity on excitation wavelength $\lambda_{\mathrm{EX}}$. PLE analysis is particularly valuable for SWNTs having PL emission in the near-IR range of $E_{11}$ transitions at excitation of the $E_{22}$ bands in visible range. From this point of view, the PL intensity maximum for sample $\mathrm{S} 1$ is located at $\lambda_{\mathrm{EM}}=999 \mathrm{~nm}$ and $\lambda_{\mathrm{EX}}=573 \mathrm{~nm}$ (Fig. 2c), correlating well with the absorption peaks for the $\mathrm{E}_{11}$ and $\mathrm{E}_{22}$ transitions of SWNTs with the $(6,5)$ chirality (curve 1 in Fig. 2a). The PL maximum of sample $S 2$ is seen in Fig. $2 d$ to be/strongly red-shifted to $\lambda_{\mathrm{EM}}=1,008 \mathrm{~nm}$ and $\lambda_{\mathrm{EX}}=575 \mathrm{~nm}$, which is much more than the slight redshift of the $\mathrm{E}_{11}$ absorption peak observed in Fig. 2a (from 999 to 1,000 nm). Besides, the small red-shift of $\lambda_{\mathrm{EX}}$ maximum is observed in Fig. 2d. The observed red shifts in PL band can be related to the formation of defects on the nanotube surface as sample S2 was ultra-sonicated at higher power.

Therefore, the results presented in Fig. 2 demonstrate that using ultra-sonication at higher power resulted in SWNTs with a higher level of defects, which is well supported by the PL spectra of samples S1 and S2 (with lower and higher defect content, respectively). Furthermore, the intensity of absorption and PL spectral peaks in Fig. 2 permit to conclude that ultra-sonication at lower and higher power resulted in different amounts of produced SWNT. More specifically, the CNT concentration in sample S2 appears to be slightly more than 2 times larger than that in sample S1. It addition, the spectra in Fig. 2 demonstrate very small amounts of CNTs with $(7,5)$ and $(8,4)$ chiralities, thus evidencing very highly efficient separation of semiconducting SWNTs through the procedure applied in this study.

\subsubsection{SWNTs as layers on solid substrate}

Annealing of the SWNTs deposited as layers onto solid substrate was found to lead to a strong reduction in their absorption and PL peaks. In Fig. 3a the PLE map of sample S2 drop-cast onto a glass slide shows a strong response of SWNTs with $(6,5)$ chirality $\left(\lambda_{\mathrm{EM}}=1,010 \mathrm{~nm}\right.$ and $\lambda_{\mathrm{EX}}=573 \mathrm{~nm}$ ), which is generally consistent with a similar map of the same sample as dispersion in liquid (Fig. 2c). After annealing at $300{ }^{\circ} \mathrm{C}$ in air for $2 \mathrm{~h}$, as well seen in Fig. $3 \mathrm{~b}$, extremely weak signals were registered. In particular, sample 
B1 (not shown here for brevity) had no PL signal in the range of $\lambda_{\mathrm{EX}}=550-600 \mathrm{~nm}$, whereas the PL signal of sample $\mathrm{B} 2$ had a small shoulder at $\lambda_{\mathrm{EX}}=580 \mathrm{~nm}$ and $\lambda_{\mathrm{EM}}=1000-1200 \mathrm{~nm}$ (Fig. 3b). It should be added that while the PLE map of sample S2 in Fig. 3a was recorded with 5-nm-wide entrance/exit slits of monochromators, the map of the annealed sample B2 (in Fig. 3b) was recorded with 14-nm-wide slits, as the PL response was strongly reduced upon annealing. After annealing, PL is seen in Fig. 3 to become extremely weak, implying transformation of isolated SWNTs into bundles and formation of BNNs with a highly quenched PL response. Nanotube bundles are known to have very weak intensities of excitonic absorption and emission [23]. Hence, our observations are in good agreement with this fact, and it is reasonable to assume that the heat-treated samples B1 and B2 are based on bundle networks.

(a)

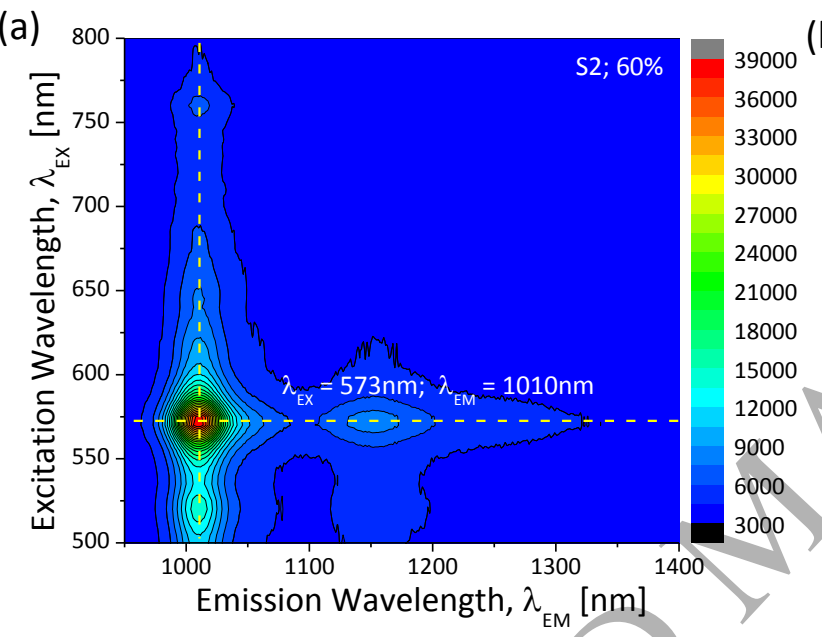

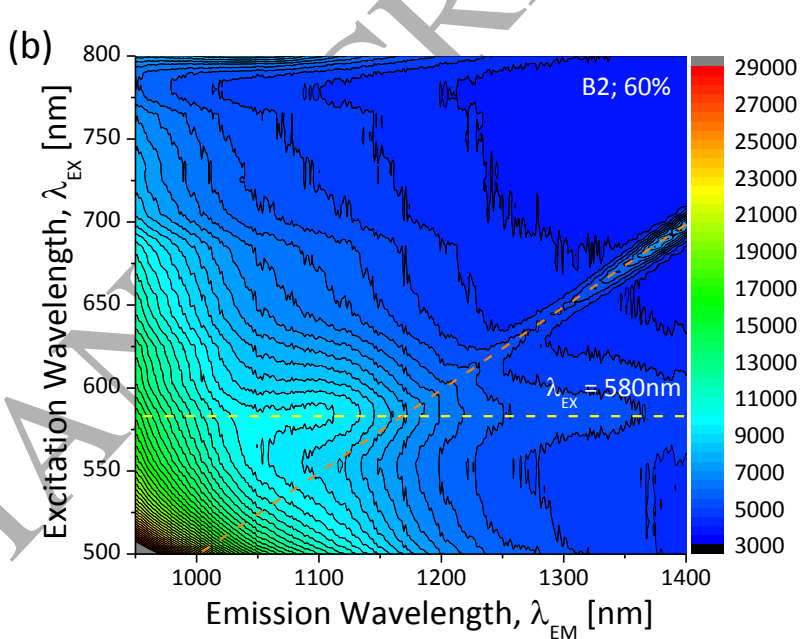

Figure 3. PLE maps of sample S2 deposited onto glass slide as a layer and measured before annealing (a) and after annealing at $300^{\circ} \mathrm{C}$ for $2 \mathrm{~h}$ (b), i.e. as sample B2. The entrance/exit slits employed in monochromators were 5 and $14 \mathrm{~nm}$, respectively, for (a) and (b).

\subsection{Electrical studies}

The as-prepared thin films of isolated nanotubes (S1 and S2) were found to have highly fluctuating resistance (from/tens of $\Omega$ to $M \Omega$ range) due to unstable percolation paths for charge carriers in thin layers of isolated nanotube networks. Good percolation could be achieved by increased thickness of the films, but, according to the recent studies, only approximately $100 \mathrm{~nm}$ of topmost surface layer contributes to $\mathrm{NH}_{3}$ sensitivity [6]. Molecules of $\mathrm{NH}_{3}$ penetrate only into a surface layer of CNT-based network where the bulk part of the films deeper than $100 \mathrm{~nm}$ is not affected by $\mathrm{NH}_{3}$. Therefore, increased thickness of SWNT-sensor layers is not considered a good approach, as bulky layer with thickness more than $100 \mathrm{~nm}$ will result in $R_{g}$ almost equal to $R_{a}$ (see formula for sensing response in section 2.5). This way, we employed alternative 
solution: to anneal SWNT+PFO-BPy films aiming at formation of bundled network of the nanotubes (BNN) with stable electrical characteristics and optimized $\mathrm{NH}_{3}$ sensitivity. After annealing, we obtained stable electrical resistance of samples B1 and B2. Regarding the different ultrasonication power applied to disperse SWNTs, the electrical resistance of samples B1 and B2 were (600 \pm 50$) \Omega$ and (3.4 \pm 0.5$) \mathrm{M} \Omega$, respectively. The difference in the resistance is attributed to high concentration of defects formed at high ultrasonication power (sample B2). To sum up, the defects decrease conductivity of the nanotubes, but result in enhancement of the $\mathrm{NH}_{3}$ sensitivity $[5,9,10]$.

\subsection{Sensing studies}

The sensing response of the S1 and S2 samples (isolated nanotubes) is merely flat towards $\sim 1,100$ ppm of used VOCs (see section 2.5). Besides, the above mentioned fluctuating resistance of isolated CNTs strongly obstructs proper measurements of sensing response of non-annealed samples consisting of highly isolated nanotubes in the polymeric matrix of PFO-BPy. For annealed samples forming BNN, B1 had a very weak sensing response, whereas sample B2 showed enhanced sensitivity for ammonia, of five volatile compounds tested (including acetone, ammonia, ethanol, isopropanol and methanol, Fig. 4). Figure 5 depicts the temporal change in the resistance of sample B2 for different concentrations of ammonia molecules. The concentration of ammonia in Fig. 5 was changed in the following way. Initially, the resistance was recorded at the ambient atmosphere for the first $60 \mathrm{~s}$. Then ammonia at the concentration of $117 \mathrm{ppm}$ was injected and retained in the bench-top test chamber during the time slot $60-210 \mathrm{~s}$. Later the ammonia was purged from the chamber by ambient air, and the process of $\mathrm{NH}_{3}$ injections were repeated at higher concentrations of 586 ppm (400-600 s), 1,172 ppm (760-900 s), 2344 ppm (1,140-1,300 s), and 2930 ppm (1,460-1630 s).

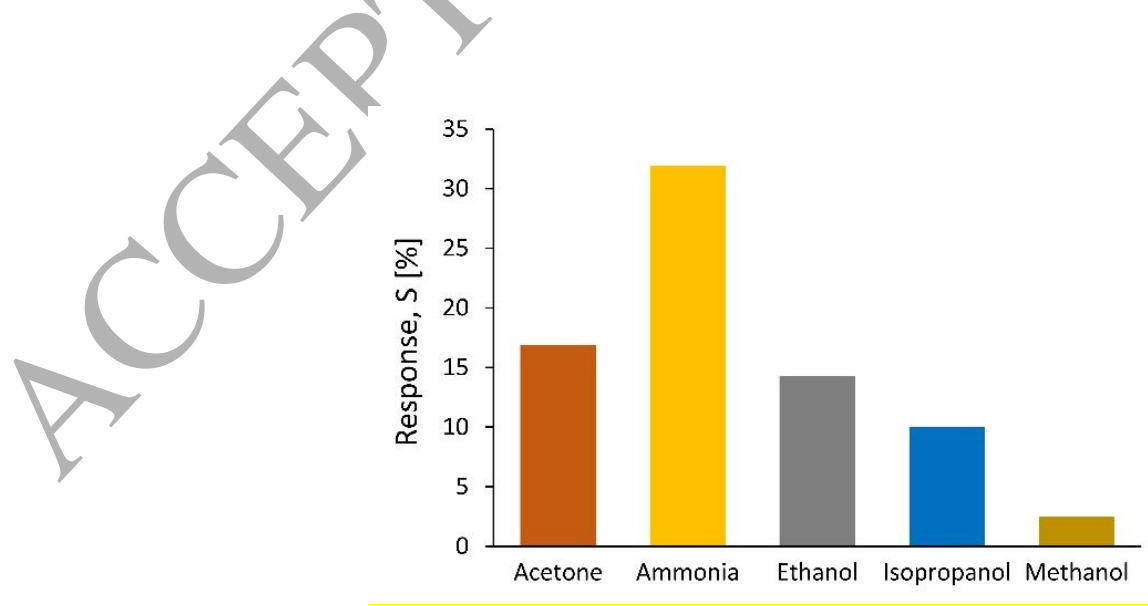

Page 9 of 15 
Figure 4. Response of sample B2 towards 1,172 ppm of acetone (17\%), ammonia (32\%), ethanol (14\%), isopropanol (10\%) and methanol (3\%).

The periodic increase and decrease of electrical resistance against successive injections and evacuations of different concentration of $\mathrm{NH}_{3}$ confirmed the adsorption-desorption chemical reaction. In Fig. $5 \mathrm{a}$, we show that the sensing layer of sample B2 effectively responds with increase of resistance for ammonia concentrations from 117 to $2,344 \mathrm{ppm}$. The sensing response, $S$, towards different concentrations of $\mathrm{NH}_{3}$ is plotted in Fig. 5b summarizes the data obtained in Fig. 4a, where the resistance values before and after $\mathrm{NH}_{3}$ injections were substituted into the Formula (1). Importantly, we observed that the concentration dependence of $S$ has a maximum in the range of 2,300-2,900 ppm. This way, the sensing response decreases for higher concentrations of ammonia. Furthermore, at certain level of ammonia, the resistance of sample B2 decreases in comparison with its initial value showing complex dynamic response. Figure 6 illustrates dramatic change in electrical resistance towards $11,700 \mathrm{ppm}$ of $\mathrm{NH}_{3}$. In general, from the Fig. 6a we observe that the baseline resistance $\left(R_{a}\right)$ of $3.2 \mathrm{M} \Omega$ reduced to $3.0 \mathrm{k} \Omega$ after the exposure to $\mathrm{NH}_{3}$ molecules $\left(R_{g}\right)$. Essentially, during this transition and immediately after $\mathrm{NH}_{3}$ exposure, there is a short time hump (peak) of an increased resistance demonstrating the initial or partial (low concentration) adsorption of $\mathrm{NH}_{3}$ on the surface of sample B2 (Fig. 6b). Later, at a bit longer exposure of approximately 20 seconds, the complete (high concertation) adsorption of $\mathrm{NH}_{3}$ led to a drastic drop in the resistance to $3.0 \mathrm{k} \Omega$. The evacuation of $\mathrm{NH}_{3}$ molecules by opening the chamber to ambient atmosphere is not resulted in the complete desorption of $\mathrm{NH}_{3}$ molecules (Fig. 6a). Because of partial desorption, the initial baseline resistance of sample B2 (3.2 M $\Omega$ ) is not attained in the ambient atmosphere. The recovery resistance was $0.66 \mathrm{M} \Omega$ being practically 5 times lower than initial baseline resistance. This process can be explained through stronger chemisorption of $\mathrm{NH}_{3}$ molecules on defect sites of CNTs at higher concentration, which implies much longer time needed for their complete desorption. Primarily, we have shown that SWNTs containing high level of defects and forming BNN due to annealing have the most prominent chemiresistive response. Chimowa et al. [10] demonstrated the preferential interaction of $\mathrm{NH}_{3}$ on the defect sites of the nanotube walls. Furthermore, sensing response of SWNTs with defects is stronger than defect-free nanotubes [9]. Thus, our results are in accord with literature data on strong influence of the nanotube defect sites on $\mathrm{NH}_{3}$ adsorption and further influence of electronic properties of the SWNTs. Our study shows that the chemiresistive sensitivity of such nanotube networks expressed as function of resistance change vs ammonia concentration is highly nonlinear characteristic. 

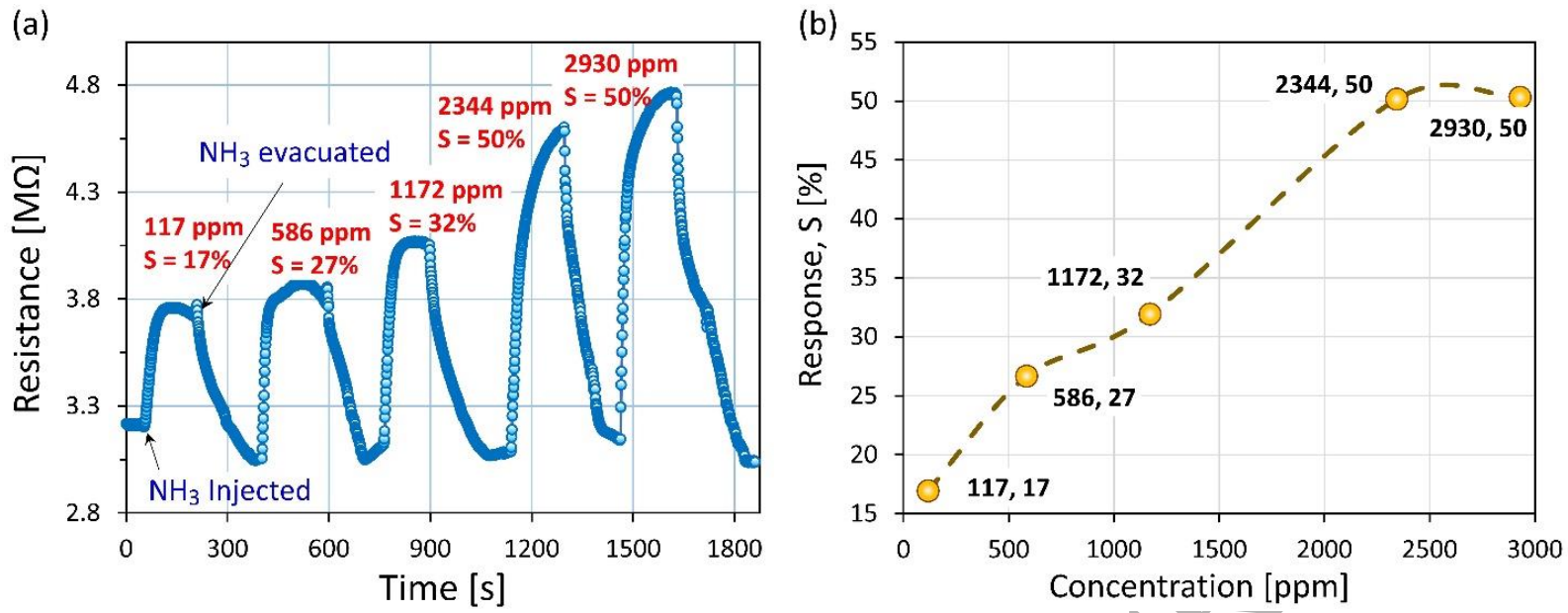

Figure 5. (a) Transient resistance response-recovery of sample $B 2$ at different concentrations of ammonia: 117 ppm (60-210 s), 586 ppm (400-600 s), 1,172 ppm (760-900 s), 2,344 ppm (1140-1300 s), and 2,930 ppm (1460-1630 s). (b) Dependence of the response (S) on the ammonia concentration in panel (a).

(a)

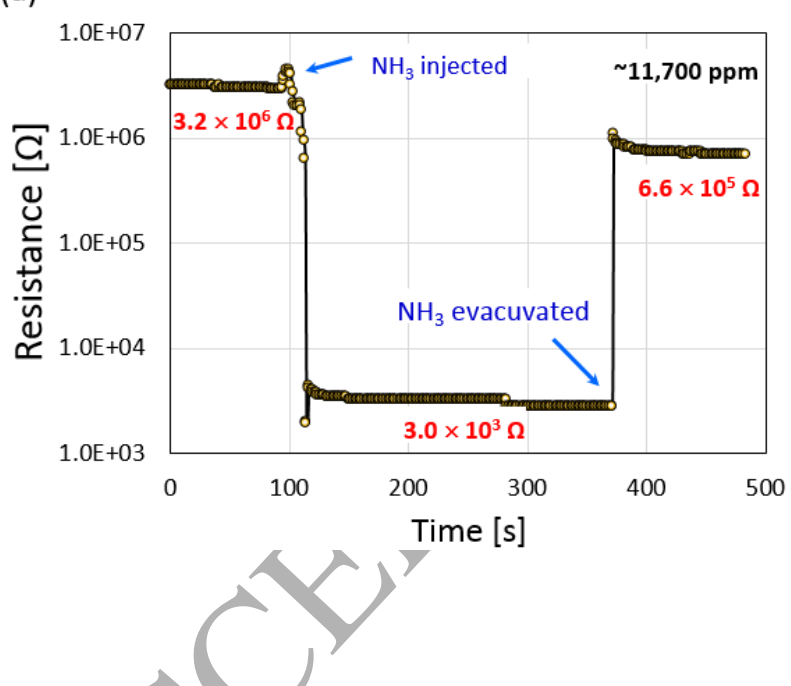

(b)

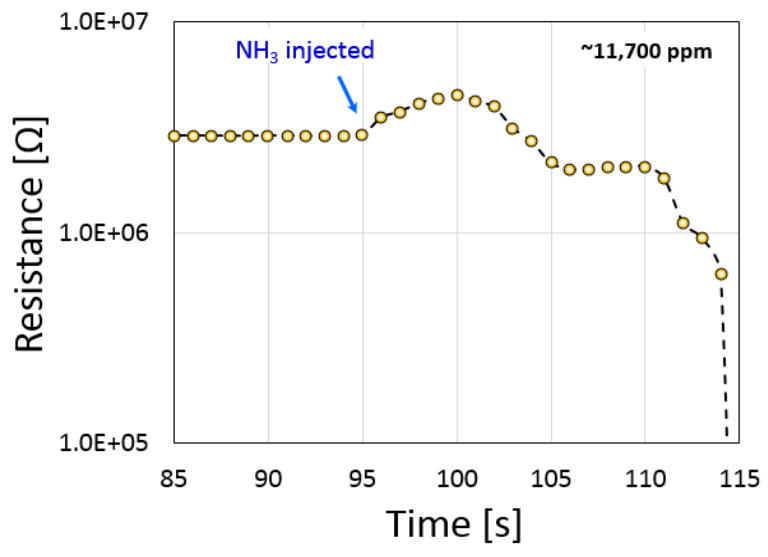

Figure 6. (a) Transient resistance response-recovery of sample $B 2$ towards $11,700 \mathrm{ppm}$ of ammonia. (b) Transient resistance response of sample B2 immediately after exposure to 11,700 ppm of ammonia.

Now we proceed with explanation of our experimental findings focusing on the knowledge on two sensing mechanisms for $\mathrm{NH}_{3}$ detection established so far. The first one is charge transfer between $\mathrm{NH}_{3}$ gas molecules and the nanotubes, and the second mechanism is the Schottky barrier modulation between nanotubes and electrodes $[4,8,25]$. In both mechanisms, semiconducting nature of the nanotubes is essential 
[4,11]. In ambient air, SWNTs are usually $p$-doped due to an interaction with oxygen molecules. For example, Bisri et al. observed ambipolar behavior of as-prepared SWNT-PFO based transistors that were converted into $p$-type unipolar devices with improved performance due to annealing in air at $400{ }^{\circ} \mathrm{C}$ [26]. Thus, the electron transfer between oxygen molecules and carbon atoms results in formation of $p$-doped semiconductor due to induction of holes [27]. Adsorption of $\mathrm{NH}_{3}$ by SWNT defects can neutralize positive charge via reduction effect and results in a rise of nanotubes resistivity. We observed the effect of increased resistance at low concentrations of ammonia. Thus, adsorption of $\mathrm{NH}_{3}$ molecules continuously increases the electron density in the conduction band compensating oxygen-induced hole density. Besides, there is another aspect of increased resistance: rise of the Schottky barrier [14]. Adsorption of $\mathrm{NH}_{3}$ molecules at the nanotube/ $\mathrm{Au}$ interface reduces work function of the $\mathrm{Au}$ electrode and the electrostatic charge balance between the nanotubes and $\mathrm{Au}$ is disturbed by the dipoles of $\mathrm{NH}_{3}$ molecules leading to an increased Schottky barrier for hole injection [14]. Thus, charge transfer between $\mathrm{NH}_{3}$ gas molecules and the nanotubes, as well as elevated Schottky barrier, can be responsible for increased resistance at both low concentrations of $\mathrm{NH}_{3}$ and initial stage with short time hump of high levels of $\mathrm{NH}_{3}$.

The results showing decreasing resistance at high Tevels of $\mathrm{NH}_{3}$ require an implication of additional mechanism in sensing process explaining this behavior. It can be rationalized in the following way. Further increase in electron density due to a higher concentration of electron donating $\mathrm{NH}_{3}$ molecules fully compensate the $p$-type conductivity of CNT, transforming them into a semiconductor with ambipolar / $n$ type dominant conductivity. The Fermi level of the metallic electrode becomes positioned closer to conduction band of the nanotubes resulting in ambipolar / $n$-type behavior with predominant contribution from electron current. Besides, it should be noted that an increase of the Schottky barrier for one type of carriers leads to reduction of the Schottky barrier height for the other [28]. In addition, the interfacial dipoles at the contact between SWNTs and Au electrode can be strongly affected by change of conductivity type of the nanotubes via adsorption-desorption of various gases [29]. This way, the described change of conductivity type for the nanotubes can be attributed to a dramatic drop of the resistance at high concentrations of electron-donor $\mathrm{NH}_{3}$ interacting with highly defective nanotube networks.

\section{Conclusions}

We have studied the mechanisms of defect induced sensing response for detection of ammonia by the networks of semiconducting carbon nanotubes. In our experiments, the best sensing has been achieved for a high-power ultrasonically-treated SWNTs that were then thermally annealed to form bundled nanotube network. We have revealed stable chemiresistive sensing with increased resistance at low concentration of 
ammonia and follow-up with dramatic drop of the resistance ( 3 orders of magnitude) at high levels of ammonia (> 2,500 ppm). To the best of our knowledge, the sensitivity via increase of carbon nanotube resistance has been studied so far for low levels of $\mathrm{NH}_{3}$. Multidirectional chemiresistive response to ammonia revealed in our experiments helped us to put forward more comprehensive mechanism of $\mathrm{NH}_{3}$ detection by carbon nanotubes. Thus, the mechanism of ammonia sensing has two major contributing phenomena. The first one is associated with a rise of nanotube resistivity due to partial neutralization of positive charge of SWNT ( $p$-type) via reduction effect of small amount of $\mathrm{NH}_{3}$. Additionally, the Schottky barrier and interface dipoles modulation at the Au/nanotube junctions can contribute to above increase of the resistance. The second phenomenon resulting in the drop of the SWNT electrical resistance is explained by full neutralization of SWNT positive charge (making the nanotube $n$-type). The consideration of the above presented comprehensive mechanism is important for better understanding of sensing behavior and further development of nanotube-based sensors of ammonia taking into account various limitations and opportunities for performance optimization.

\section{Acknowledgments}

P.M.L., A.G.R. and S.A.K. would like to thank the Daiwa Anglo-Japanese Foundation (Daiwa Foundation Award 11425/12174) for supporting this work. P.M.L. and A.G.R. acknowledge support of the Royal Academy of Engineering / The Leverhulme Trust (Senior Research Fellowship, no. LTSRF1617/13/57). Finally, S.A.K. wishes to thank the Japan Society for the Promotion of Science (JSPS, grant no. 16K04904).

Page 13 of 15 


\section{References}

[1] M.S. Dresselhaus, G. Dresselhaus, P. Avouris, Carbon Nanotubes: Synthesis, Structure, Properties, and Applications, Springer-Verlag, Berlin - Heidelberg, 2001.

[2] R.S. Lee, H.J. Kim, J.E. Fischer, A. Thess, R.E. Smalley, Conductivity enhancement in singlewalled carbon nanotube bundles doped with $\mathrm{K}$ and $\mathrm{Br}$, Nature 388 (1997) 255-257. https://doi.org/10.1038/40822.

[3] S. Seppälä, E. Häkkinen, M.J. Alava, V. Ermolov, E.T. Seppälä, Electrical transport properties of percolating random networks of carbon nanotube bundles, EPL Europhys. Lett. 91 (2010) 47002. https://doi.org/10.1209/0295-5075/91/47002.

[4] J. Kong, N.R. Franklin, C. Zhou, M.G. Chapline, S. Peng, K. Cho, H. Dai, Nanotube molecular

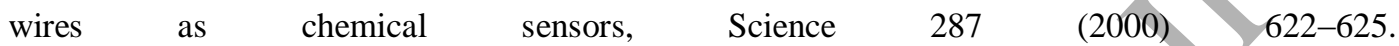
https://doi.org/10.1126/science.287.5453.622.

[5] J.A. Robinson, E.S. Snow, Ş.C. Bădescu, T.L. Reinecke, F.K. Perkins, Role of defects in singlewalled carbon nanotube chemical sensors, Nano Lett. 6 (2006) 1747-1751. https://doi.org/10.1021/n10612289.

[6] E. Bekyarova, I. Kalinina, M.E. Itkis, L. Beer, N. Cabrera, R.C. Haddon, Mechanism of ammonia detection by chemically functionalized single-walled carbon nanotubes: in situ electrical and optical study of gas analyte detection, J. Am. Chem. Soc. 129 (2007) 10700-10706. https://doi.org/10.1021/ja0703271.

[7] H.M. Tóháti, Á. Pekker, B. Pataki, Z. Szekrényes, K. Kamarás, Bundle versus network conductivity of carbon nanotubes separated by typé, Eur. Phys. J. B 87 (2014) 1-6. https://doi.org/10.1140/epjb/e2014-41103-9.

[8] F. Rigoni, S. Freddi, S. Pagliara, G. Drera, L. Sangaletti, J.M. Suisse, M. Bouvet, A.M. Malovichko, A.V. Emelianov, I.I. Bobrinetskiy, Humidity-enhanced sub-ppm sensitivity to ammonia of covalently functionalized single-wall carbon nanotube bundle layers, Nanotechnology 28 (2017) 255502. https://doi.org/10.1088/1361-6528/aa6da7.

[9] J. Kim, S.W. Choi, J.H. Lee, Y. Chung, Y.T. Byun, Gas sensing properties of defect-induced single-walled carbon nanotubes, Sens. Actuators B 228 (2016) 688-692. https://doi.org/10.1016/j.snb.2016.01.094.

[10] G. Chimowa, B. Matsoso, N.J. Coville, S.S. Ray, E. Flahaut, T. Hungria, L. Datas, B.W. Mwakikunga, Preferential adsorption of NH3 gas molecules on MWCNT defect sites probed using in situ Raman spectroscopy, Phys. Status Solidi 214 (2017) 1600930. https://doi.org/10.1002/pssa.201600930.

[11] S.G. Wang, Q. Zhang, D.J. Yang, P.J. Sellin, G.F. Zhong, Multi-walled carbon nanotube-based gas sensors for NH3 detection, Diam. Relat. Mater. 13 (2004) 1327-1332. https://doi.org/10.1016/j.diamond.2003.11.070.

[12] M.Y. Timmermans, D. Estrada, A.G. Nasibulin, J.D. Wood, A. Behnam, D. ming Sun, Y. Ohno, J.W. Lyding, A. Hassanien, E. Pop, E.I. Kauppinen, Effect of carbon nanotube network morphology on thin film transistor performance, Nano Res. 5 (2012) 307-319. https://doi.org/10.1007/s12274-012-0211-8.

[13] D. Hecht, L. Hu, G. Grüner, Conductivity scaling with bundle length and diameter in single walled carbon nanotube networks, Appl. Phys. Lett. 89 (2006) 1-4. https://doi.org/10.1063/1.2356999.

[14] N. Peng, Q. Zhang, C.L. Chow, O.K. Tan, N. Marzari, Sensing mechanisms for carbon nanotube based NH3 gas detection, Nano Lett. 9 (2009) 1626-1630. https://doi.org/10.1021/nl803930w.

[15] S. Chopra, K. McGuire, N. Gothard, A.M. Rao, A. Pham, Selective gas detection using a carbon 
nanotube sensor, Appl. Phys. Lett. 83 (2003) 2280-2282. https://doi.org/10.1063/1.1610251.

[16] J. Suehiro, G. Zhou, M. Hara, Fabrication of a carbon nanotube-based gas sensor using dielectrophoresis and its application for ammonia detection by impedance spectroscopy, J. Phys. D: Appl. Phys. 36 (2003) L109-L114. https://doi.org/10.1088/0022-3727/36/21/L01.

[17] A. Graf, Y. Zakharko, S.P. Schießl, C. Backes, M. Pfohl, B.S. Flavel, J. Zaumseil, Large scale, selective dispersion of long single-walled carbon nanotubes with high photoluminescence quantum yield by shear force mixing, Carbon 105 (2016) 593-599. https://doi.org/10.1016/j.carbon.2016.05.002.

[18] H. Ozawa, N. Ide, T. Fujigaya, Y. Niidome, N. Nakashima, One-pot separation of highly enriched (6,5)-single-walled carbon nanotubes using a fluorene-based copolymer, Chem. Lett. 40 (2011) 239-241. https://doi.org/10.1246/cl.2011.239.

[19] Y. Pan, H.K.F. Cheng, L. Li, S.H. Chan, J. Zhao, Y.K. Juay, Annealing induced electrical conductivity jump of multi-walled carbon nanotube/polypropylene composites and influence of molecular weight of polypropylene, J. Polym. Sci. B 48 (2010) 2238-2247. https://doi.org/10.1002/polb.22106.

[20] W. Li, Y. Zhang, J. Yang, J. Zhang, Y. Niu, Z. Wang, Thermal annealing induced enhancements of electrical conductivities and mechanism for multiwalled carbon nanotubes filled poly(ethyleneco -hexene) composites, ACS Appl. Mater. Interfaces 4 (2012) 6468-6478. https://doi.org/10.1021/am302597f.

[21] P. Shankar, J.B.B. Rayappan, Racetrack effect on the dissimilar sensing response of $\mathrm{ZnO}$ thin film - an anisotropy of isotropy, ACS Appl. Mater. Interfaces 8 (2016) 24924-24932. https://doi.org/10.1021/am302597f.

[22] P. Lutsyk, Y. Piryatinski, M. Alaraimi, R. Arif, M. Shandura, O. Kachkovsky, A. Verbitsky, A. Rozhin, Emergence of additional visible-range photoluminescence due to aggregation of cyanine dye: astraphloxin on carbon nanotubes dispersed with anionic surfactant, J. Phys. Chem. C 120 (2016) 20378-20386. https://doi.org/10.1021/acs.jpcc.6b06272.

[23] P.H. Tan, A.G. Rozhin, T. Hasan, P. Hu, V. Scardaci, W.I. Milne, A.C. Ferrari, Photoluminescence spectroscopy of carbon nanotube bundles: evidence for exciton energy transfer. Phys. Rev. Lett. 99 (2007) 137402. https://doi.org/10.1103/PhysRevLett.99.137402.

[24] M. Kastner, S. Stahl, I. Vollert, C. Loi, N. Rühl, T. Hertel, F. Schöppler, A comparison of Raman and photoluminescence spectra for the assessment of single-wall carbon nanotube sample quality, Chem. Phys. Lett. 635 (2015) 245-249. https://doi.org/10.1016/j.cplett.2015.06.076.

[25] Y. Battie, L. Gorintin, O. Ducloux, P. Thobois, P. Bondavalli, G. Feugnet, A. Loiseau, Thickness dependent sensing mechanism in sorted semi-conducting single walled nanotube based sensors, Analyst 137 (2012) 2151-2157. https://doi.org/10.1039/c2an15949j.

[26] S.Z. Bisri, J/Gao, V. Derenskyi, W. Gomulya, I. Iezhokin, P. Gordiichuk, A. Herrmann, M.A. Loi, High performance ambipolar field-effect transistor of random network carbon nanotubes, Adv. Mater. 24 (2012) 6147-6152. https://doi.org/10.1002/adma.201202699.

[27] D. Kang, N. Park, J. Ko, E. Bae, W. Park, Oxygen-induced p-type doping of a long individual single-walled carbon nanotube, Nanotechnology $16 \quad$ (2005) 1048-1052. https://doi.org/10.1088/0957-4484/16/8/008.

[28] J. Svensson, E.E.B. Campbell, Schottky barriers in carbon nanotube-metal contacts, J. Appl. Phys. 110 (2011) 111101. https://doi.org/10.1063/1.3664139.

[29] X. Cui, M. Freitag, R. Martel, L. Brus, P. Avouris, Controlling energy-level alignments at carbon nanotube/Au contacts, Nano Lett. 3 (2003) 783-787. https://doi.org/10.1021/n1034193a. 